\title{
A supersymmetric color superconductor from holography
}

\author{
Antón F. Faedo, ${ }^{a}$ David Mateos, ${ }^{a, b}$ Christiana Pantelidou ${ }^{c}$ and Javier Tarrío ${ }^{d}$ \\ ${ }^{a}$ Departament de Física Quántica i Astrofísica and Institut de Ciències del Cosmos (ICC), \\ Universitat de Barcelona, \\ Martí i Franquès 1, ES-08028, Barcelona, Spain \\ ${ }^{b}$ Institució Catalana de Recerca i Estudis Avançats (ICREA), \\ Passeig Lluís Companys 23, ES-08010, Barcelona, Spain \\ ${ }^{c}$ Centre for Particle Theory and Department of Mathematical Sciences, Durham University, \\ Durham, DH1 3LE, U.K. \\ ${ }^{d}$ Physique Théorique et Mathématique, Université Libre de Bruxelles (ULB), \\ and International Solvay Institutes, \\ Campus de la Plaine CP 231, B-1050, Brussels, Belgium \\ E-mail: afaedo@ffn.ub.es, dmateos@icrea.cat, \\ christiana.pantelidou@durham.ac.uk, tarrio@gmail.com
}

AbStract: We use holography to study $d=4, \mathcal{N}=4, \mathrm{SU}\left(N_{\mathrm{c}}\right)$ super Yang-Mills coupled to $N_{\mathrm{f}} \ll N_{\mathrm{c}}$ quark flavors. We place the theory at finite isospin density $n_{\mathrm{I}}$ by turning on an isospin chemical potential $\mu_{\mathrm{I}}=M_{\mathrm{q}}$, with $M_{\mathrm{q}}$ the quark mass. We also turn on two R-symmetry charge densities $n_{1}=n_{2}$. We show that the ground state is a supersymmetric, superfluid, color superconductor, namely a finite-density state that preserves a fraction of supersymmetry in which part of the global symmetries and part of the gauge symmetries are spontaneously broken. The holographic description consists of $N_{\mathrm{f}} \mathrm{D} 7$-brane probes in $\mathrm{AdS}_{5} \times \mathrm{S}^{5}$. The symmetry breaking is due to the dissolution of some D3-branes inside the D7-branes triggered by the electric field associated to the isospin charge. The massless spectrum contains Goldstone bosons and their fermionic superpartners. The massive spectrum contains long-lived, mesonic quasi-particles if $n_{\mathrm{I}} \ll \mu_{\mathrm{I}}^{3}$, and no quasi-particles otherwise. We discuss the possibility that, despite the presence of mass scales and charge densities in the theory, conformal and relativistic invariance arise as emergent symmetries in the infrared.

KEYWORDS: AdS-CFT Correspondence, Gauge-gravity correspondence

ARXiv EPrint: 1807.09712 


\section{Contents}

1 Introduction 1

2 Model 2

3 Higgs branch 3

4 Solution 4

5 Physical interpretation 5

6 Symmetry breaking $\quad 6$

$\begin{array}{lll}7 & \text { Spectrum } & 7\end{array}$

8 Discussion $\quad 7$

\section{Introduction}

Quantum Chromodynamics (QCD) at non-zero baryon density $n_{\mathrm{b}}$ is notoriously difficult to analyze. Because of asymptotic freedom, the preferred phase at asymptotically high density can be shown to be a color-flavor locked (CFL) configuration $[1,2]$ (for a review see [3]). The ground state in this regime is a color superconductor, namely a finite-density state in which the color symmetry is Higgsed. Following a common abuse of language, we will refer to this as the spontaneous breaking of the color symmetry. In addition, the CFL ground state is also a superfluid, since the baryon number symmetry is spontaneously broken too. In the regime of high but finite density, such as at the core of neutron stars, no first-principle calculations are possible. The only non-perturbative tool, namely lattice QCD, is of limited applicability due to the so-called sign problem [4].

This situation provides one motivation to study the physics of QCD as some other conserved charge is taken to be large, for example the isospin charge. In this case the sign problem is absent and the theory can be simulated on the lattice (see e.g. [5]). Analytical methods can also be used $[6,7]$. The emergent picture is that the ground state is a superfluid with superfluidity driven by a pion condensate at low density and by a quark-antiquark condensate at high density. No color superconductivity was found in these analysis.

In this paper we give a step towards the holographic description of color superconducting phases. In this context the goal is not to do precision physics but to perform first-principle calculations that may lead to interesting insights [8]. In the case of QCD at non-zero temperature, the insights obtained through this program range from static properties to far-from-equilibrium dynamics of strongly coupled plasmas (see e.g. [9] and references therein). 
We will investigate a simple yet extremely rich holographic model which exhibits both color superconductivity and superfluidity when a certain combination of conserved charges is taken to be large. Our model differs from QCD in many respects, including the fact that it is supersymmetric, that it exhibits no chiral symmetry and hence no pions, and that it possesses an R-symmetry that is absent in QCD. Therefore we do not claim that our results have any direct implications for real-world QCD. However, we believe that they are interesting for three reasons. First, they show that color superconductivity does appear in holography when some conserved charges are large (in this case a combination of isospin charge and R-charge). Second, we expect that a similar holographic mechanism will give rise to color superconductivity in the presence of baryon density [10]. Third, to the best of our knowledge our model is the first example of a supersymmetric color superconductor. Color superconductivity in supersymmetric theories has been previously considered in e.g. [11-13], but in these cases all the supersymmetries are broken by the ground state. In contrast, in our model the ground state leaves some supersymmetry unbroken. We expect that this property will facilitate a precise comparison between the strong-coupling limit described by holography and the weak-coupling regime accessible via perturbative field theory methods.

Color superconductivity in the holographic context has been previously explored. Refs. [14, 15] considered baryon density instead of isospin density, ref. [16] studied a bottomup model instead of a top-down model, and refs. $[17,18]$ mimicked the breaking of the color symmetry as the breaking of a global symmetry.

\section{Model}

Type IIB string theory on the near-horizon geometry of $N_{\mathrm{c}}$ D3-branes and $N_{\mathrm{f}}$ D7-branes is dual to $d=4, \mathcal{N}=4, \mathrm{SU}\left(N_{\mathrm{c}}\right)$ super Yang-Mills theory coupled to $N_{\mathrm{f}}$ hypermultiplets in the fundamental representation. The presence of the hypermultiplets breaks supersymmetry to $\mathcal{N}=2$, so we will refer to this theory simply as "the $\mathcal{N}=2$ gauge theory". Although the hypermultiplets contain both bosons and fermions, we will loosely refer to them as "flavors" or "quarks". In the regime $N_{\mathrm{f}} \ll N_{\mathrm{c}}$ the D7-branes can be treated as probes [19] in the $\mathrm{AdS}_{5} \times \mathrm{S}^{5}$ geometry

$$
\mathrm{d} s^{2}=H^{-\frac{1}{2}}\left(-\mathrm{d} t^{2}+\mathrm{d} \vec{x}^{2}\right)+H^{\frac{1}{2}}\left(\mathrm{~d} y_{i}^{2}+\mathrm{d} z_{\alpha}^{2}\right),
$$

where $t, \vec{x}$ are the four gauge theory directions parallel to the D3-branes, $y^{i}$ with $i=1, \ldots, 4$ are the coordinates along the D7-branes orthogonal to the D3-branes, and $z^{\alpha}$ with $\alpha=1,2$ are the coordinates orthogonal to both sets of branes. We will often write the metric in the $y^{i}$ directions in spherical coordinates as

$$
\mathrm{d} y_{i}^{2}=d r^{2}+r^{2}\left(\omega_{1}^{2}+\omega_{2}^{2}+\omega_{3}^{2}\right)
$$

where $\omega_{n}$ are the left-invariant forms on $\mathrm{S}^{3} . H$ is the usual harmonic function in the six-dimensional space transverse to the D3-branes:

$$
H=\frac{L^{4}}{\left(r^{2}+z_{1}^{2}+z_{2}^{2}\right)^{2}},
$$

with $L$ the radius of $\mathrm{AdS}_{5}$ and $\mathrm{S}^{5}$. 
The dynamics of the $N_{\mathrm{f}}$ D7-branes may be described by the non-Abelian action of [20]. At the lowest order in the string tension this reduces to a super-Yang-Mills-Higgs (SYMH) action together with extra couplings to background fluxes coming from the Wess-Zumino term. As emphasised by the author himself, the action in [20] is known to be incomplete, but it seems to capture the exact physics for supersymmetric configurations [21,22]. In fact, supersymmetric solutions of the SYMH action often become solutions of the full action. This is also the case here [23], and therefore we will effectively work with the SYMH action. For simplicity we will focus on the case $N_{\mathrm{f}}=2$ and we will refer to the two flavors as $u$ and $d$ quarks.

In the background (2.1) the SYMH action takes the form

$$
\begin{aligned}
\frac{S}{T_{\mathrm{D} 7}}= & -\int \frac{1}{2} \operatorname{Tr}\left(F \wedge * F+H^{\frac{1}{2}} \delta_{\alpha \beta} D Z^{\alpha} \wedge * D Z^{\beta}\right) \\
& -\int \frac{1}{2} H^{-1} \mathrm{~d} t \wedge \mathrm{d}^{3} x \wedge \operatorname{Tr}(F \wedge F) .
\end{aligned}
$$

Throughout this paper we set $2 \pi \ell_{s}^{2}=1$, so all quantities are effectively dimensionless. The last term in (2.4) comes from the coupling to the $\mathrm{RR}$ five-form that supports the geometry (2.1). $F$ is the $\mathrm{U}(2)_{\mathrm{f}}$ non-Abelian field strength on the world volume of the D7branes and $D$ is the gauge covariant derivative. $Z^{\alpha}$ are non-Abelian scalars (Higgs fields) in the adjoint of $\mathrm{U}(2)_{\mathrm{f}}$, which parametrize the (in general non-commuting) positions of the D7-branes in the $z^{\alpha}$-plane. The Hodge dual $*$ is taken with respect to the eight-dimensional induced metric on the branes, $g$. The solutions that we will consider will all lie within the $\mathrm{SU}(2)_{\mathrm{f}}$ subgroup of $\mathrm{U}(2)_{\mathrm{f}}=\mathrm{SU}(2)_{\mathrm{f}} \times \mathrm{U}(1)_{\mathrm{b}}$ and will be translationally-invariant along the $\vec{x}$-directions. Therefore we will be effectively studying $\mathrm{SU}(2)_{\mathrm{f}}$ configurations in the five dimensions $\{t, \vec{y}\}$. The $\mathrm{U}(1)_{\mathrm{b}}$ charge can be thought of as the baryon number and will play no role here.

\section{Higgs branch}

The $\mathcal{N}=2$ gauge theory possesses a continuous moduli space of vacua parametrized by the vacuum expectation values (VEVs) of (s)quark bilinear operators. Supersymmetry guarantees that all the ground states in this so-called Higgs branch are exactly degenerate. ${ }^{1}$ Holographic studies of the Higgs branch include [24-27]. In these papers no baryon or isospin density was considered.

At a generic vacuum on the Higgs branch part of the $\mathrm{SU}\left(N_{\mathrm{c}}\right)$ gauge symmetry is spontaneously broken (some global symmetries are broken too, see section 6). At weak coupling in the gauge theory the breaking can be seen by analysing the part of the $\mathrm{SU}\left(N_{\mathrm{c}}\right)$ symmetry that is broken by a specific set of VEVs in a fixed gauge (see e.g. [24]). At strong coupling the breaking is due to the separation of some D3-branes from the others, which gives a mass to the strings stretching between them. Since these strings are dual to the gluons in the gauge theory this signals the breaking of the $\mathrm{SU}\left(N_{\mathrm{c}}\right)$ gauge group. The

\footnotetext{
${ }^{1}$ There is also a Coulomb branch parametrized by the VEVs of the adjoint scalars of the theory.
} 
separated D3-branes dissolve inside the D7-branes and appear as instantons of the SYMH theory on the D7-branes. ${ }^{2}$

The breaking can also be seen explicitly by considering the backreaction of the D7branes-plus-instanton on the spacetime fields. This results in a position-dependent RR five-form flux [31] and therefore in a scale-dependent effective rank of the gauge group. The size of the instanton $\Lambda$ is dual to the scale of gauge symmetry breaking and, in the absence of charge densities, it is arbitrary. A key point of our paper is that, in the presence of isospin and R-charge densities, the scale of gauge symmetry breaking is fixed by the charges. In other words, in the absence of charge densities one may place the theory at an arbitrary point on the Higgs branch and thus break the gauge symmetry "by hand". In contrast, in the presence of charges the system is driven to a specific point on the Higgs branch fixed by the charges and the gauge symmetry is broken dynamically.

\section{Solution}

We consider a direct importation of the dyonic instanton solution of [32]

$$
\begin{aligned}
A & =a_{t}(r) \mathrm{d} t \otimes \sigma^{3}+a(r) \delta_{m n} \omega^{n} \otimes \sigma^{n}, \\
Z^{1} & =Z=\phi(r) \sigma^{3}, \quad Z^{2}=0,
\end{aligned}
$$

where $\sigma^{n}$ are the Pauli matrices. We split the field strength into purely electric and purely magnetic parts: $F=\mathrm{d} t \wedge E+F_{\text {mag. }}$. If the following first-order BPS conditions are satisfied

$$
F_{\text {mag }}=-\star F_{\text {mag }}, \quad E=-D Z,
$$

where the Hodge dual $\star$ is taken with respect to the flat metric along the $y$-directions, then the configuration (4.1) preserves $\mathcal{N}=1$ supersymmetry and it solves the second-order equations of motion of SYMH theory in flat space [32]. The first equation is the usual (anti)selfduality condition associated to instantonic configurations, whereas the second one relates the electric field and the scalar and, via Gauss' law, implies that $D \star D Z=0$. As expected from supersymmetry, this configuration also solves the equations of motion of the full action for a pair of D-branes [20] in flat space [33]. Crucially, the same is true for a pair of D7-branes placed in the background (2.1) [23].

The solution of the BPS equations of interest to us is

$$
a(r)=\frac{\Lambda^{2}}{r^{2}+\Lambda^{2}}, \quad a_{t}(r)=\phi(r)=\frac{M_{\mathrm{q}} r^{2}}{r^{2}+\Lambda^{2}},
$$

where $\Lambda$ and $M_{\mathrm{q}}$ are integration constants. The solution is completely regular everywhere. The asymptotic form

$$
a_{t}(r)=\phi(r) \simeq M_{\mathrm{q}}-\frac{M_{\mathrm{q}} \Lambda^{2}}{r^{2}}+\cdots
$$

will be useful below.

\footnotetext{
${ }^{2}$ This is a particular example of the more general phenomenon of "Branes within branes" [28, 29] whereby a low-dimensional brane dissolves inside a high-dimensional brane. From the viewpoint of the effective field theory on the high-dimensional brane, the low-dimensional brane appears as a flux of the worldvolume gauge field. A pedagogical discussion can be found in [30]. In the context of holography only the case of an instanton flux on the D7-branes leads to color superconductivity because this is the case where the dissolved objects are precisely the D3-branes associated to the color symmetry of the dual gauge theory.
} 


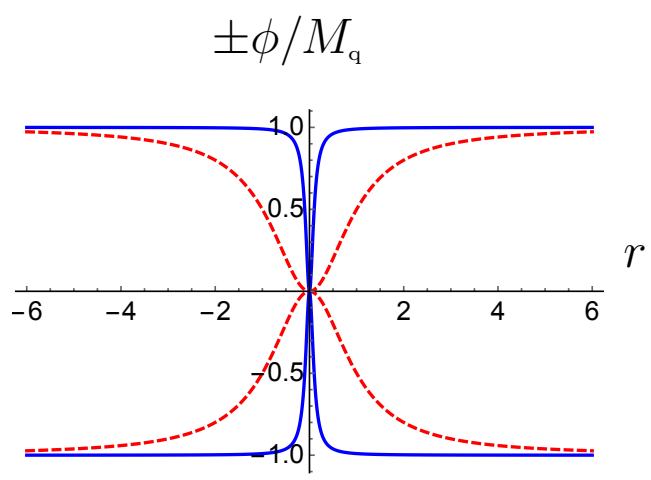

Figure 1. Brane profiles for $\Lambda=1$ (dashed, red curve) and $\Lambda=1 / 10$ (solid, blue curve).

Choosing the scalar to point in the $\sigma^{3}$ direction explicitly breaks the $\mathrm{SU}(2)_{\text {f }}$ gauge symmetry down to $\mathrm{U}(1)_{\mathrm{f}}$. This allows us to define the electric charge of the instanton by projecting onto the unbroken $\mathrm{U}(1)_{\mathrm{f}}$ as

$$
q=\lim _{r \rightarrow \infty} \frac{1}{M_{\mathrm{q}}} \int_{S^{3}} r^{3} \operatorname{Tr}\left(Z E_{r}\right)=2 \pi^{2} \Lambda^{2} M_{\mathrm{q}},
$$

where the integral is taken on a three-sphere of radius $r$ in the metric (2.2). Despite the explicit breaking of $\mathrm{SU}(2)_{\mathrm{f}}$ to an Abelian subgroup, the instanton is prevented from collapse by the non-zero angular momentum produced by the crossed electric and magnetic nonAbelian fields [34]. The Poynting momentum density is aligned with the $\omega^{3}$ left-invariant form in (2.2), which results in a self-dual angular momentum with equal skew-eigenvalues in the $y^{i}$-directions $n_{1}=n_{2} \propto q$.

\section{Physical interpretation}

The fact that $Z(r)$ is proportional to $\sigma^{3}$, which is diagonal with entries \pm 1 , means that the branes bend in opposite directions along the $z^{1}$-axis with otherwise identical profiles, as shown in figure 1. The asymptotic behaviour (4.4) has two immediate consequences. First, the constant $M_{\mathrm{q}}$ corresponds to the quark mass [19]. To be precise, the quark mass is a complex number and we see that the masses of the $u$ and $d$ quarks are equal in magnitude but have opposite phases. The same is true for the corresponding quark condensates

$$
\langle\bar{u} u\rangle=-\langle\bar{d} d\rangle \propto-M_{\mathrm{q}} \Lambda^{2} .
$$

Note that this corresponds to a vanishing chiral condensate

$$
\langle\bar{\Psi} \Psi\rangle=\langle\bar{u} u+\bar{d} d\rangle=0
$$

but to a nonzero condensate of the form

$$
\left\langle\bar{\Psi} \sigma^{3} \Psi\right\rangle=\langle\bar{u} u-\bar{d} d\rangle \propto-M_{\mathrm{q}} \Lambda^{2} .
$$

Second, the isospin chemical potential and the charge densities are given by $[35,36]$

$$
\mu_{\mathrm{I}}=M_{\mathrm{q}}, \quad n_{\mathrm{I}}=n_{\mathrm{u}}=-n_{\mathrm{d}} \propto-M_{\mathrm{q}} \Lambda^{2} .
$$


From the viewpoint of the dual gauge theory, the angular momentum corresponds to equal R-charge densities [37-39]

$$
n_{\mathrm{R}} \equiv n_{1}=n_{2}
$$

along two of the three $\mathrm{U}(1)$ factors in the Cartan subalgebra of the $\mathrm{SO}(6)$ R-symmetry of $\mathcal{N}=4$ SYM. Note that all the charge densities are comparable since

$$
n_{\mathrm{I}} \sim n_{\mathrm{R}} \sim q .
$$

Moreover, the size of the instanton is not a free parameter but is fixed as

$$
\Lambda^{2} \propto \frac{n_{\mathrm{R}}}{\mu_{\mathrm{I}}}
$$

In summary, the solution (4.3) represents a state in the $\mathcal{N}=2$ gauge theory with non-zero isospin and R-charge densities related to one another and to the quark mass through (5.4), (5.5) and (5.6). The $\mathcal{N}=2$ gauge theory has been previously studied both at non-zero isospin density [35, 40, 41] and at non-zero baryon density [14, 36, 42-44]. In these papers no supersymmetric ground state was identified.

\section{$6 \quad$ Symmetry breaking}

Four-dimensional $\mathcal{N}=4 \mathrm{SYM}$ is invariant under conformal transformations and possesses an SO(6) R-symmetry. Coupling the theory to massive quarks has several effects. First, it breaks conformal invariance. Second, it decreases the supersymmetry to $\mathcal{N}=2$. Third, it reduces the $\mathrm{SO}(6)$ symmetry to $\mathrm{SO}(4)=\mathrm{SU}(2)_{\mathrm{L}} \times \mathrm{SU}(2)_{\mathrm{R}}$, where $\mathrm{SU}(2)_{\mathrm{R}}$ is the R-symmetry of the $\mathcal{N}=2$ algebra and $\mathrm{SU}(2)_{\mathrm{L}}$ is a global symmetry that does not act on the $\mathcal{N}=2$ supercharges. For massless quarks there would be an extra $\mathrm{U}(1)_{\mathrm{R}}$ factor. The addition of $N_{\mathrm{f}}=2$ quark flavors of equal mass would introduce a new $\mathrm{SU}(2)_{\mathrm{f}} \times \mathrm{U}(1)_{\mathrm{b}}$ global symmetry that would rotate the quarks into one another. If the quark masses differ even just in phase, as in our case, then this symmetry is explicitly broken to $\mathrm{U}(1)_{\mathrm{f}} \times \mathrm{U}(1)_{\mathrm{b}}$.

On the gravity side these breakings can be understood geometrically. The original $\mathrm{SO}(6)$ symmetries are the isometries of the $\mathrm{S}^{5}$. The addition of the D7-branes selects a four-plane in the $\mathbb{R}^{6}$ space transverse to the D3-branes and thus reduces the symmetry to $\mathrm{SO}(4)$. The $\mathrm{SU}(2)_{\mathrm{L}}$ factor leaves each $\omega_{n}$ form in $(2.2)$ invariant, whereas the $\mathrm{SU}(2)_{\mathrm{R}}$ rotates them into one another. For massless quarks the branes lie at the origin of the $z^{12}$-plane and an additional $\mathrm{U}(1)_{\mathrm{R}}$ corresponding to rotations in this plane is preserved. Instead, a non-zero quark mass breaks the $\mathrm{U}(1)_{\mathrm{R}}$ explicitly. The $\mathrm{SU}(2)_{\mathrm{f}} \times \mathrm{U}(1)_{\mathrm{b}}$ global symmetry of the gauge theory becomes the non-Abelian gauge symmetry on the pair of D7-branes, which is broken explicitly to $\mathrm{U}(1)_{\mathrm{f}} \times \mathrm{U}(1)_{\mathrm{b}}$ by the fact that the branes bend in opposite direction in the $z^{1}$-axis.

In summary, $\mathcal{N}=4 \mathrm{SYM}$ coupled to massive quarks of unequal masses is invariant under an $\mathrm{SU}\left(N_{\mathrm{c}}\right)$ gauge symmetry and an $\mathrm{SU}(2)_{\mathrm{L}} \times \mathrm{SU}(2)_{\mathrm{R}} \times \mathrm{U}(1)_{\mathrm{f}} \times \mathrm{U}(1)_{\mathrm{b}}$ global symmetry. Placing the theory at non-zero density by turning on $n_{\mathrm{I}}$ and $n_{\mathrm{R}}$ results in the spontaneous breaking of some of these symmetries. This can be easily seen on the gravity side. First, the presence of an instanton indicates that some D3-branes have dissolved 
inside the D7-branes. As explained in section 3 this implies that the gauge group is spontaneously broken, with the scale of the breaking given by (5.7). Second, the self-dual angular momentum generated by the simultaneous presence of the isospin electric field and the instanton spontaneously breaks part of the global symmetries as $\mathrm{SU}(2)_{\mathrm{R}} \times \mathrm{U}(1)_{\mathrm{f}} \rightarrow \mathrm{U}(1)_{\mathrm{D}}$, where the group on the right-hand side is the diagonal U(1) in the Cartan subalgebra of the left-hand side. This breaking can be seen geometrically as follows. The fact that the Poynting vector distinguishes between $\omega^{3}$ and $\omega^{1,2}$ breaks $\mathrm{SU}(2)_{\mathrm{R}}$ to the $\mathrm{U}(1)_{\mathrm{R}}$ subgroup that rotates $\omega^{1,2}$ into one another. The fact that only simultaneous rotations of $\omega^{n}$ and $\sigma^{n}$ leave the second term in (4.1a) invariant then breaks $\mathrm{U}(1)_{\mathrm{R}} \times \mathrm{U}(1)_{\mathrm{f}}$ to the diagonal $\mathrm{U}(1)_{\mathrm{D}}$. This is similar to the breaking of $\mathrm{SU}(2)_{\mathrm{R}} \times \mathrm{SU}(2)_{\mathrm{f}}$ to $\mathrm{SU}(2)_{\mathrm{D}}$ that takes place on the Higgs branch in the absence of the electric field (see e.g. [24]).

\section{Spectrum}

Since several global symmetries are broken spontaneously, we expect the spectrum to contain massless bosons. Given that the ground state preserves $\mathcal{N}=1$ supersymmetry, we expect them to be accompanied by the corresponding massless, fermionic superpartners. The fact that the ground state breaks Lorentz invariance means that the number of these modes need not coincide with the number of broken generators [45, 46]. Holographic examples of this phenomenon include [47-49]. We will report on the massless modes elsewhere [23].

The qualitative properties of the spectrum of massive modes depend on the value of the ratio $\epsilon=\Lambda^{2} / M_{\mathrm{q}}^{2} \propto n_{\mathrm{I}} / \mu_{\mathrm{I}}^{3}$. For small $\epsilon$ the spectrum contains long-lived, mesonic quasi-particles; otherwise no massive quasi-particles are present. This is illustrated in figure 2, where we show the spectral function at zero spatial momentum for the gauge theory operator dual to the $\sigma^{3}$ component of the scalar field $Z^{2}$, calculated via standard methods [50] from fluctuations governed by the action (2.4). For small $\epsilon$ we see high and narrow peaks associated to long-lived excitations, which on the gravity side correspond to quasi-normal modes (QNM) of the $Z^{2}$ field on the D7-branes with very small imaginary parts compared to their real parts. The position of these peaks agrees almost exactly with the masses of mesons on a single D7-brane with the same value of $M_{\mathrm{q}}$ [51]. For $\epsilon=1$ no such peaks are present. This feature is straightforward to understand on the gravity side. As seen in figure 1, for small $\epsilon$ the branes' profiles approach those of [51] everywhere except on a very thin throat that connects the branes to the Poincaré horizon at the origin of AdS. The imaginary part of the QNMs measures the absorption probability by the horizon. Since this vanishes as the throat closes off, in this limit the imaginary parts of the QNMs frequencies vanish and the real parts converge to the real frequencies of [51].

\section{Discussion}

We have considered $d=4, \mathcal{N}=4, \mathrm{SU}\left(N_{\mathrm{c}}\right)$ super Yang-Mills coupled to $N_{\mathrm{f}} \ll N_{\mathrm{c}}$ quarks, which breaks the supersymmetry to $\mathcal{N}=2$. We have placed the theory at non-zero isospin and R-charge densities. We have shown that, if the conditions (5.4) and (5.5) are obeyed, then the ground state of the system is a supersymmetric, superfluid, color superconductor. 


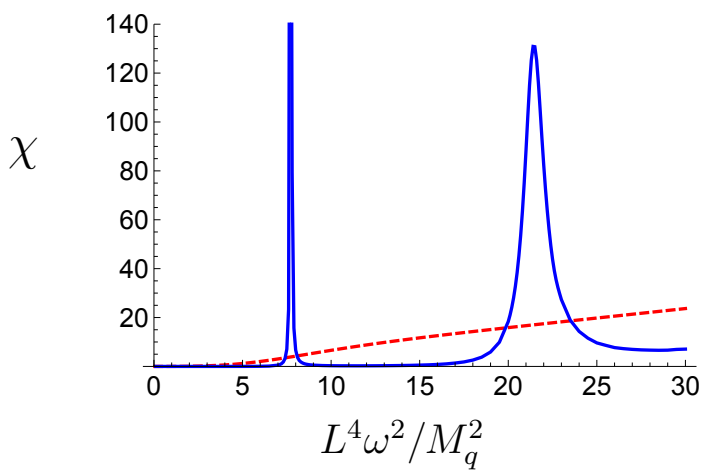

Figure 2. Spectral function described in the text (with arbitrary normalization) for $\epsilon=1$ (dashed, red curve) and $\epsilon=1 / 100$ (solid, blue curve).

The solution that we considered is a unit-charge instanton centered at $r=0$. In this case the scale of gauge symmetry breaking is fixed by the charge densities. This is an essential difference with the physics of the Higgs branch ${ }^{3}$ in which this scale is arbitrary.

Solutions for the case $N_{\mathrm{f}}>2$ with any instanton number are straightforward to construct because multidyonic instanton solutions for higher-rank gauge groups are known [34]. For these configurations the possible symmetry-breaking patterns are richer [23].

The superconducting property in our system is fundamentally different from that in what are usually referred to as "holographic superconductors", in which the broken symmetry is actually a global symmetry [53-55]. In contrast, in our case the broken color symmetry is a strongly coupled, non-Abelian gauge symmetry. In our probe approximation this breaking is encoded in the dissolution of some D3-brane charge on the D7-branes in the form of an instanton. The fact that the instanton size is completely fixed by the asymptotic charges proves that the Fermi seasickness [56] that causes the dissolution of the D3-branes in the first place need not result in a runaway potential. Were we to include the backreaction of the D7-branes-plus-instanton on spacetime then the color breaking would be visible in the running of the color gauge group, as in [31]. In this backreacted scenario one would also be able to work in the grand-canonical ensemble for the R-charges. Instead, we are limited to the canonical ensemble because the gauge fields whose asymptotic values would allow us to define the R-charge chemical potentials are off-diagonal components of the ten-dimensional metric, whose dynamics is frozen in our probe approximation.

To the best of our knowledge our solution is the first example of a supersymmetric color superconductor. The BPS equations (4.2) are crucial to prove that the solution of the SYMH equations is also a solution of the full non-Abelian action of [20]. It would be interesting to investigate whether the full open string equations of motion are also satisfied, as in [57].

Supersymmetry requires the isospin to be critical, $\mu_{\mathrm{I}}=M_{\mathrm{q}}$, and the R-charges to be equal, $n_{1}=n_{2}$. It would be interesting to relax these conditions both on the gravity side and on the field theory side. In the case of near-critical values it may be possible to compare a weak-coupling field-theory analysis along the lines of $[58,59]$ with the strong-coupling holographic result.

\footnotetext{
${ }^{3}$ And in a certain approximation in the presence of a baryon density [52].
} 
We have referred to our system as a superfluid because it breaks some global symmetries spontaneously. However, this does not directly imply that the system is able to support a superflow. It would be interesting to investigate this along the lines of e.g. [60].

In our solution the scalar and the time-component of the gauge field behave as

$$
\phi=a_{t}=0+\mathcal{O}\left(r^{2}\right)
$$

in the IR. The fact that the scalar field vanishes implies that the induced metric on the D7-branes in the IR is $\operatorname{AdS}_{5} \times \mathrm{S}^{3}$. The $\mathrm{AdS}_{5}$ factor suggests that the IR physics may be conformally invariant despite the presence of several mass scales in the theory. The vanishing of the electric field suggests that relativistic invariance may also be restored in the IR despite the presence of non-zero charge densities. These speculations are under investigation [23].

\section{Acknowledgments}

We thank Stefano Carignano, Roberto Emparan, Bartomeu Fiol, Eduardo Fraga, Jaume Garriga, Prem Kumar, and very specially Carlos Hoyos, for discussions. We are grateful to Jorge Casalderrey-Solana for a critical reading of the manuscript. AF and DM are supported by grants FPA2016-76005-C2-1-P, FPA2016-76005-C2-2-P, 2014-SGR-104, 2014-SGR-1474, SGR-2017-754 and MDM-2014-0369. CP is supported by the STFC Consolidated Grant ST/P000371/1. JT is supported by the Advanced ARC project "Holography, Gauge Theories and Quantum Gravity" and by the Belgian Fonds National de la Recherche Scientifique FNRS (convention IISN 4.4503.15).

Open Access. This article is distributed under the terms of the Creative Commons Attribution License (CC-BY 4.0), which permits any use, distribution and reproduction in any medium, provided the original author(s) and source are credited.

\section{References}

[1] M.G. Alford, K. Rajagopal and F. Wilczek, QCD at finite baryon density: Nucleon droplets and color superconductivity, Phys. Lett. B 422 (1998) 247 [hep-ph/9711395] [INSPIRE].

[2] M.G. Alford, K. Rajagopal and F. Wilczek, Color flavor locking and chiral symmetry breaking in high density QCD, Nucl. Phys. B 537 (1999) 443 [hep-ph/9804403] [InSPIRE].

[3] M.G. Alford, A. Schmitt, K. Rajagopal and T. Schäfer, Color superconductivity in dense quark matter, Rev. Mod. Phys. 80 (2008) 1455 [arXiv:0709.4635] [InSPIRE].

[4] P. de Forcrand, Simulating QCD at finite density, PoS(LAT2009) 010 (2009) [arXiv: 1005.0539] [INSPIRE].

[5] J.B. Kogut and D.K. Sinclair, The Finite temperature transition for 2-flavor lattice QCD at finite isospin density, Phys. Rev. D 70 (2004) 094501 [hep-lat/0407027] [INSPIRE].

[6] D.T. Son and M.A. Stephanov, QCD at finite isospin density, Phys. Rev. Lett. 86 (2001) 592 [hep-ph/0005225] [INSPIRE]. 
[7] D.T. Son and M.A. Stephanov, QCD at finite isospin density: From pion to quark-antiquark condensation, Phys. Atom. Nucl. 64 (2001) 834 [hep-ph/0011365] [INSPIRE].

[8] D. Mateos, Gauge/string duality applied to heavy ion collisions: Limitations, insights and prospects, J. Phys. G 38 (2011) 124030 [arXiv:1106.3295] [INSPIRE].

[9] J. Casalderrey-Solana, H. Liu, D. Mateos, K. Rajagopal and U.A. Wiedemann, Gauge/String Duality, Hot QCD and Heavy Ion Collisions, Cambridge University Press (2014) [arXiv: 1101.0618] [INSPIRE].

[10] A.F. Faedo, D. Mateos, C. Pantelidou and J. Tarrio, work in progress.

[11] R. Harnik, D.T. Larson and H. Murayama, Supersymmetric color superconductivity, JHEP 03 (2004) 049 [hep-ph/0309224] [INSPIRE].

[12] M. Arai and N. Okada, Color superconductivity in $N=2$ supersymmetric gauge theories, Phys. Rev. D 74 (2006) 045004 [hep-th/0512234] [INSPIRE].

[13] B.S. Rajput and S. Kumar, Color superconductivity in supersymmetric gauge theories, Int. J. Theor. Phys. 50 (2011) 1342 [INSPIRE].

[14] H.-Y. Chen, K. Hashimoto and S. Matsuura, Towards a Holographic Model of Color-Flavor Locking Phase, JHEP 02 (2010) 104 [arXiv:0909.1296] [INSPIRE].

[15] M. Rozali, D. Smyth and E. Sorkin, Holographic Higgs Phases, JHEP 08 (2012) 118 [arXiv: 1202.5271] [INSPIRE].

[16] P. Basu, F. Nogueira, M. Rozali, J.B. Stang and M. Van Raamsdonk, Towards A Holographic Model of Color Superconductivity, New J. Phys. 13 (2011) 055001 [arXiv:1101.4042] [INSPIRE].

[17] K. Bitaghsir Fadafan, J. Cruz Rojas and N. Evans, Holographic description of color superconductivity, Phys. Rev. D 98 (2018) 066010 [arXiv: 1803.03107] [INSPIRE].

[18] K. Ghoroku, K. Kashiwa, Y. Nakano, M. Tachibana and F. Toyoda, Color Superconductivity in Holographic SYM Theory, arXiv:1902.01093 [inSPIRE].

[19] A. Karch and E. Katz, Adding flavor to AdS/CFT, JHEP 06 (2002) 043 [hep-th/0205236] [INSPIRE].

[20] R.C. Myers, Dielectric branes, JHEP 12 (1999) 022 [hep-th/9910053] [INSPIRE].

[21] A. Hashimoto, The Shape of branes pulled by strings, Phys. Rev. D 57 (1998) 6441 [hep-th/9711097] [INSPIRE].

[22] D. Bak, J.-H. Lee and H. Min, Dynamics of BPS states in the Dirac-Born-Infeld theory, Phys. Rev. D 59 (1999) 045011 [hep-th/9806149] [INSPIRE].

[23] A.F. Faedo, D. Mateos, C. Pantelidou and J. Tarrio, Spectrum of a supersymmetric color superconductor, to appear.

[24] J. Erdmenger, J. Grosse and Z. Guralnik, Spectral flow on the Higgs branch and AdS/CFT duality, JHEP 06 (2005) 052 [hep-th/0502224] [INSPIRE].

[25] Z. Guralnik, S. Kovacs and B. Kulik, Holography and the Higgs branch of $N=2 S Y M$ theories, JHEP 03 (2005) 063 [hep-th/0405127] [INSPIRE].

[26] Z. Guralnik, Strong coupling dynamics of the Higgs branch: Rolling a Higgs by collapsing an instanton, Nucl. Phys. B 732 (2006) 46 [hep-th/0412074] [INSPIRE]. 
[27] Z. Guralnik, S. Kovacs and B. Kulik, AdS/CFT duality and the Higgs branch of $N=2 S Y M$, Fortsch. Phys. 53 (2005) 480 [hep-th/0501154] [INSPIRE].

[28] E. Witten, Bound states of strings and p-branes, Nucl. Phys. B 460 (1996) 335 [hep-th/9510135] [INSPIRE].

[29] M.R. Douglas, Branes within branes, NATO Sci. Ser. C 520 (1999) 267 [hep-th/9512077] [INSPIRE].

[30] C.V. Johnson, D-brane primer, in Strings, branes and gravity. Proceedings, Theoretical Advanced Study Institute, TASI'99, Boulder, U.S.A., May 31-June 25, 1999, pp. 129-350 (2000) [DOI:10.1142/9789812799630_0002] [hep-th/0007170] [INSPIRE].

[31] A.F. Faedo, D. Mateos, C. Pantelidou and J. Tarrio, Unquenched flavor on the Higgs branch, JHEP 11 (2016) 021 [arXiv: 1607.07773] [INSPIRE].

[32] N.D. Lambert and D. Tong, Dyonic instantons in five-dimensional gauge theories, Phys. Lett. B 462 (1999) 89 [hep-th/9907014] [INSPIRE].

[33] M. Zamaklar, Geometry of the nonAbelian DBI dyonic instanton, Phys. Lett. B 493 (2000) 411 [hep-th/0006090] [INSPIRE].

[34] E. Eyras, P.K. Townsend and M. Zamaklar, The Heterotic dyonic instanton, JHEP 05 (2001) 046 [hep-th/0012016] [INSPIRE].

[35] R. Apreda, J. Erdmenger, N. Evans and Z. Guralnik, Strong coupling effective Higgs potential and a first order thermal phase transition from AdS/CFT duality, Phys. Rev. D 71 (2005) 126002 [hep-th/0504151] [INSPIRE].

[36] S. Kobayashi, D. Mateos, S. Matsuura, R.C. Myers and R.M. Thomson, Holographic phase transitions at finite baryon density, JHEP 02 (2007) 016 [hep-th/0611099] [INSPIRE].

[37] S.S. Gubser, Thermodynamics of spinning D3-branes, Nucl. Phys. B 551 (1999) 667 [hep-th/9810225] [INSPIRE].

[38] A. Chamblin, R. Emparan, C.V. Johnson and R.C. Myers, Charged AdS black holes and catastrophic holography, Phys. Rev. D 60 (1999) 064018 [hep-th/9902170] [InSPIRE].

[39] M. Cvetič and S.S. Gubser, Phases of $R$ charged black holes, spinning branes and strongly coupled gauge theories, JHEP 04 (1999) 024 [hep-th/9902195] [INSPIRE].

[40] J. Erdmenger, M. Kaminski and F. Rust, Isospin diffusion in thermal AdS/CFT with flavor, Phys. Rev. D 76 (2007) 046001 [arXiv:0704.1290] [inSPIRE].

[41] J. Erdmenger, M. Kaminski, P. Kerner and F. Rust, Finite baryon and isospin chemical potential in AdS/CFT with flavor, JHEP 11 (2008) 031 [arXiv:0807.2663] [INSPIRE].

[42] A. Karch and A. O'Bannon, Metallic AdS/CFT, JHEP 09 (2007) 024 [arXiv:0705.3870] [INSPIRE].

[43] A. Karch and A. O'Bannon, Holographic thermodynamics at finite baryon density: Some exact results, JHEP 11 (2007) 074 [arXiv:0709.0570] [INSPIRE].

[44] D. Mateos, S. Matsuura, R.C. Myers and R.M. Thomson, Holographic phase transitions at finite chemical potential, JHEP 11 (2007) 085 [arXiv:0709.1225] [INSPIRE].

[45] B.I. Halperin, Dynamic properties of the multicomponent Bose fluid, Phys. Rev. B 11 (1975) 178. 
[46] H.B. Nielsen and S. Chadha, On How to Count Goldstone Bosons, Nucl. Phys. B 105 (1976) 445 [INSPIRE].

[47] V.G. Filev, C.V. Johnson and J.P. Shock, Universal Holographic Chiral Dynamics in an External Magnetic Field, JHEP 08 (2009) 013 [arXiv:0903.5345] [INSPIRE].

[48] I. Amado, D. Arean, A. Jimenez-Alba, K. Landsteiner, L. Melgar and I.S. Landea, Holographic Type II Goldstone bosons, JHEP 07 (2013) 108 [arXiv:1302.5641] [INSPIRE].

[49] R. Argurio, A. Marzolla, A. Mezzalira and D. Naegels, Note on holographic nonrelativistic Goldstone bosons, Phys. Rev. D 92 (2015) 066009 [arXiv:1507.00211] [INSPIRE].

[50] D.T. Son and A.O. Starinets, Minkowski space correlators in AdS/CFT correspondence: Recipe and applications, JHEP 09 (2002) 042 [hep-th/0205051] [INSPIRE].

[51] M. Kruczenski, D. Mateos, R.C. Myers and D.J. Winters, Meson spectroscopy in AdS/CFT with flavor, JHEP 07 (2003) 049 [hep-th/0304032] [INSPIRE].

[52] M. Ammon, K. Jensen, K.-Y. Kim, J.N. Laia and A. O'Bannon, Moduli Spaces of Cold Holographic Matter, JHEP 11 (2012) 055 [arXiv: 1208.3197] [INSPIRE].

[53] S.S. Gubser, Breaking an Abelian gauge symmetry near a black hole horizon, Phys. Rev. D 78 (2008) 065034 [arXiv:0801.2977] [INSPIRE].

[54] S.A. Hartnoll, C.P. Herzog and G.T. Horowitz, Building a Holographic Superconductor, Phys. Rev. Lett. 101 (2008) 031601 [arXiv:0803.3295] [INSPIRE].

[55] S.A. Hartnoll, C.P. Herzog and G.T. Horowitz, Holographic Superconductors, JHEP 12 (2008) 015 [arXiv:0810.1563] [INSPIRE].

[56] S.A. Hartnoll, J. Polchinski, E. Silverstein and D. Tong, Towards strange metallic holography, JHEP 04 (2010) 120 [arXiv:0912.1061] [INSPIRE].

[57] L. Thorlacius, Born-Infeld string as a boundary conformal field theory, Phys. Rev. Lett. 80 (1998) 1588 [hep-th/9710181] [INSPIRE].

[58] T.J. Hollowood, S.P. Kumar, A. Naqvi and P. Wild, $N=4 S Y M$ on $S^{3}$ with Near Critical Chemical Potentials, JHEP 08 (2008) 046 [arXiv:0803.2822] [INSPIRE].

[59] T.J. Hollowood, S.P. Kumar and J.C. Myers, Weak coupling large- $N$ transitions at finite baryon density, JHEP 11 (2011) 138 [arXiv:1110.0696] [INSPIRE].

[60] I. Amado, D. Areán, A. Jiménez-Alba, K. Landsteiner, L. Melgar and I. Salazar Landea, Holographic Superfluids and the Landau Criterion, JHEP 02 (2014) 063 [arXiv: 1307.8100] [INSPIRE]. 